\title{
Inelastic Neutron Scattering Studies on the Crystal Field Excitations in Superconducting $\mathrm{NdFeAsO}_{0.85} \mathrm{~F}_{0.15}$
}

\author{
Peng Cheng,, Wei Bao, ${ }^{1}$ Sergey Danilkin, ${ }^{2}$ Tiesong Zhao, ${ }^{1}$ Jieming Sheng, \\ Juanjuan Liu, ${ }^{1}$ Wei Luo, ${ }^{1}$ and Jinchen Wang ${ }^{1}$ \\ ${ }^{1}$ Department of Physics, Renmin University of China, Beijing 100872, China \\ ${ }^{2}$ Bragg Institute, Australian Nuclear Science and Technology Organization, Locked Bag 2001, Kirrawee DC, NSW 2232, Australia \\ Correspondence should be addressed to Peng Cheng; pcheng@ruc.edu.cn
}

Received 7 December 2014; Revised 26 February 2015; Accepted 2 March 2015

Academic Editor: Viorel Sandu

Copyright (C) 2015 Peng Cheng et al. This is an open access article distributed under the Creative Commons Attribution License, which permits unrestricted use, distribution, and reproduction in any medium, provided the original work is properly cited.

Inelastic neutron scattering experiments were performed on polycrystalline samples of $\mathrm{NdFe} \mathrm{AsO}_{0.85} \mathrm{~F}_{0.15}$ over a wide temperature range $(3 \mathrm{~K}-250 \mathrm{~K})$. Based on the analysis of the experimental data, a $\mathrm{Nd}^{3+} \mathrm{CF}$ energy level scheme is proposed to give a consistent explanation about the observed CF transitions. The observed extra ground-state CF transitions could not be simply explained by the transitions between five Kramers doublets split from the $\mathrm{Nd}^{3+}{ }^{4} I_{9 / 2}$ ground state in the $C_{4 v}$ point symmetry. A reliable explanation would be a superposition of crystal fields due to different local symmetries around the $\mathrm{Nd}^{3+}$ ions induced by the fluorine doping.

\section{Introduction}

For materials with rare-earth $(\mathrm{R})$ elements, the interactions between f-electrons and the crystal field potentials produced by local lattice environment play important roles in determining the electronic ground state of $\mathrm{R}$ ions, as well as in understanding the magnetic and thermodynamic properties of these compounds. Inelastic neutron scattering has been widely accepted as the most reliable technique in determining the crystal field potentials in rare-earth based materials. Particularly, for superconducting materials, in either high- $T_{c}$ cuprates or other R based heavy fermion superconductors, important research progress has been made in investigating the neutron spectroscopy of the CF excitations $[1,2]$.

The discovery of Fe-based superconductor $\mathrm{LaFe}^{\mathrm{AsO}} \mathrm{O}_{1-x}$ $\mathrm{F}_{x}$ has caused a tremendous upsurge of scientific interest since 2008 [3]. Shortly after the report of $26 \mathrm{~K}$ superconductivity in $\mathrm{LaFeAsO}_{1-x} \mathrm{~F}_{x}$, through the substitution of $\mathrm{La}$ by other $\mathrm{R}$ elements with f-electrons (such as $\mathrm{Ce}, \mathrm{Pr}, \mathrm{Nd}$, and $\mathrm{Sm}$ ), superconducting critical temperature $T_{c}$ above $50 \mathrm{~K}$ could be observed in $\mathrm{RFeAsO}_{1-x} \mathrm{~F}_{x}$ [4-6]. So far, these so-called FeAs-1111 compounds mark the highest $T_{c}$ in all Fe-based superconductors, which also provided a unique opportunity in studying the correlation between CF excitations and physical properties in these exotic superconducting families. Up to now, there have been several publications about crystal field studies in FeAs-1111 compounds through inelastic neutron scattering technique. For example, in $\mathrm{CeFeAsO}_{1-x} \mathrm{~F}_{x}(x=0,0.16)$, the Ce CF levels were determined and the intrinsic linewidth $\Gamma$ of $18.7 \mathrm{meV}$ was found to have a clear anomaly at $T_{c}$ [7]; inelastic neutron scattering measurements of $\mathrm{CF}$ transitions in $\mathrm{RFeAsO}_{1-x} \mathrm{~F}_{x}(\mathrm{RE}=\mathrm{Pr}$, $\mathrm{Nd}$ ) provided evidences that there were two distinct charge environments around the $\mathrm{RE}$ ions arising from a random distribution of fluorine ions [8]. The CF level structures for both paramagnetic phase and antiferromagnetic phase of $\mathrm{NdFeAsO}$ were constructed by Xiao et al. [9]. However, for $\mathrm{NdFeAsO} \mathrm{O}_{1-x} \mathrm{~F}_{x}$, there are still insufficient neutron scattering data on the CF transitions and the CF energy level scheme of $\mathrm{Nd}$ ion is still not clear.

In this paper, we report inelastic neutron scattering studies on superconducting $\mathrm{NdFeAsO}_{0.85} \mathrm{~F}_{0.15}$ samples. Based on the analysis of inelastic neutron scattering spectra at different temperatures, we propose a crystal field energy level scheme for $\mathrm{Nd}$ ion, which could explain the $\mathrm{CF}$ transitions observed in the experimental data. The additional CF transitions beyond the requirements of $C_{4 v}$ point symmetry indicate the superposition of crystal field 
and inhomogeneous ligand environments around the rareearth ions in $\mathrm{NdFeAsO}_{0.85} \mathrm{~F}_{0.15}$.

\section{Experimental Results and Discussions}

About 20 grams of $\mathrm{NdFeAsO}_{0.85} \mathrm{~F}_{0.15}$ polycrystalline samples were synthesized using the solid state reaction method. Resistivity measurements show that the onset superconducting transition temperature of our samples is about $47 \mathrm{~K}$ while the state of zero resistivity is reached at about $43 \mathrm{~K}$, as shown in the inset of Figure 1(a). Inelastic neutron scattering measurements on $\mathrm{NdFeAsO}_{0.85} \mathrm{~F}_{0.15}$ were carried out on thermal triple-axis spectrometer TAIPAN at the Australian research reactor OPAL [10]. The final neutron energy $E_{f}$ was set to be $14.7 \mathrm{meV}$, with pyrolytic graphite (PG) as the monochromator and analyzer. The collimation was $48^{\prime}-40^{\prime}$ $40^{\prime}-120^{\prime}$.

Figure 1 shows the raw data of the inelastic neutron spectra for $\mathrm{NdFeAsO}_{0.85} \mathrm{~F}_{0.15}$ at different temperatures. From $3 \mathrm{~K}$ to $250 \mathrm{~K}$, constant-Q scans were performed every 50 Kelvin. The momentum transfer $Q$ was fixed to be $3.1 \AA$ in the whole measurements. First of all, the strong peaks at about $45 \mathrm{meV}$ and $40 \mathrm{meV}$ (marked with red stars) could be easily distinguished as the spurious peaks, which originated from the $2 \lambda$ elastic neutron scattering. (The best energy windows for PG filter are $14.7 \mathrm{meV}$ and $13.5 \mathrm{meV}$. In our experimental setup, $E_{f}$ was fixed to be $14.7 \mathrm{meV}$; when $E_{i}=58.8 \mathrm{meV}$ and $54 \mathrm{meV}$, the energy transfers of $2 \lambda$ neutron (14.7 meV and $13.5 \mathrm{meV}$ ) were corresponding to the strong elastic scattering signals. That is the origin of the spurious peaks at the energy transfers equaling $44.1 \mathrm{meV}$ and $40.5 \mathrm{meV}$.) At below $30 \mathrm{meV}$ plenty of CF excitation peaks could be clearly observed in the spectra at $3 \mathrm{~K}$ (marked with green arrows). With increasing temperature, the intensities of these peaks gradually decreased, which indicates that the corresponding CF excitations are ground-state excitations. In high temperature regions, some new CF peaks gradually emerge at around $11 \mathrm{meV}$ and their absolute intensities got higher with increasing temperature. So these peaks should correspond to the excitations between excited CF states.

In order to fit and carefully analyze the CF peaks, after subtracting the spurious peaks, the next thing needed is to clarify the phonon contributions from the inelastic neutron spectrum. From both experimental data and theoretical calculations, it is known that the phonon spectra are almost the same for both parent compound and F-doped superconductors in FeAs-1111 system [11-13]. So with the generalized phonon density of states (GDOS) of $\mathrm{NdFeAsO}$ in reference paper [9], after transformation with Bose factor and the neutron cross section equation $[14,15]$

$$
\frac{d^{2} \sigma}{d \Omega d E}=N \frac{k_{f}}{k_{i}} \frac{\left(\gamma r_{0}\right)^{2}}{4} g_{J}^{2} S(Q, E),
$$

we can finally calculate the phonon background for each temperature in our inelastic neutron spectra. The results calculated for $T=3 \mathrm{~K}$ are shown in Figure 1(f) (red hollow scatters).
After subtracting phonons, we noticed that the spectrum at $3 \mathrm{~K}$ almost overlaps with the spectrum at high temperatures in energy transfer above $36 \mathrm{meV}$ except for notable additional intensity contributions between $40 \mathrm{meV}$ and $48 \mathrm{meV}$. These additional neutron intensities gradually disappeared with increasing temperatures, which indicates the existence of ground-state CF excitations in this energy range. So it is reasonable to choose the spectrum above $36 \mathrm{meV}$ at high temperature as instrument background because these intensities could not come from CF excitations and most likely result from the $2 \lambda$ neutron contributions. After subtracting both the phonon and instrument background, the spectrum obtained at $3 \mathrm{~K}$ could be well fitted by seven $\mathrm{CF}$ excitation peaks with the method of Gaussian fitting, as shown in Figure 2(a) (labelled as A-G, resp.). The similar crystal field excitations at energy transfer below $30 \mathrm{meV}$ were also observed by Goremychkin et al. [8]. Because our data included higher energy transfer region, we could observe the CF excitations at $41.5 \mathrm{meV}$ and $44.5 \mathrm{meV}$. From both Figures 1 and 2(b), it can be seen that the intensities of all these seven CF peaks decrease with increasing temperatures, which is clearly the characteristic of ground-state excitations.

Therefore based on our inelastic neutron data, we proposed a $\mathrm{Nd}^{3+} \mathrm{CF}$ energy level scheme for $\mathrm{NdFeAsO}_{0.85} \mathrm{~F}_{0.15}$, which is illustrated in Figure 3. The crystal field energy levels of $\mathrm{NdFeAsO}$ were obtained from another publication [9]. As shown in Figure 3, under crystal field with the $C_{4 v}$ point symmetry, the $\mathrm{Nd}^{3+}{ }^{4} I_{9 / 2}$ ground state splits into five doublets in $\mathrm{NdFeAsO}$ at temperatures above the antiferromagnetic and structural phase transition [9]. For $\mathrm{NdFeAsO}_{0.85} \mathrm{~F}_{0.15}$, the ground-state CF transitions marked as " $\mathrm{A}$ " to " $\mathrm{G}$ " in Figure 3 were observed in the inelastic neutron spectra as shown in both Figures 1 and 2(a). From the comparison between the $150 \mathrm{~K}$ and $3 \mathrm{~K}$ data in Figure 2(b), we could also notice that, in high temperature neutron spectra, there were some new $\mathrm{CF}$ peaks emerging at around $11 \mathrm{meV}$ and $26 \mathrm{meV}$. These new $\mathrm{CF}$ peaks were corresponding to the excitations between excited states, as illustrated in Figure 3 (i.e., transitions " $\mathrm{H}$ " and "I"). The consistence between the observed CF transitions and the CF energy level scheme proved the validity of our CF model proposed in this paper.

It is clear that the CF level scheme of $\mathrm{NdFeAsO}_{0.85} \mathrm{~F}_{0.15}$ is significantly different from that of tetragonal $\mathrm{NdFeAsO}$. In order to understand this difference, first of all we need to check whether there were CF states splitting caused by magnetic phase transitions. In the inset of Figure 1(a), the typical temperature dependence of resistances was measured on our samples. There were no traces of phase transitions because there was no anomaly in the normal state resistance which should be the typical features in the samples with structural or magnetic phase transitions. The resistance measurements were also performed in different batches of our samples which show similar features. Also based on our previous neutron measurements on samples with similar doping [16], we could safely rule out the possibility of structural or magnetic phases transitions induced additional CF states.

All the CF peaks should correspond to certain transitions of $\mathrm{Nd}$ f-electrons between different $\mathrm{CF}$ states. In 


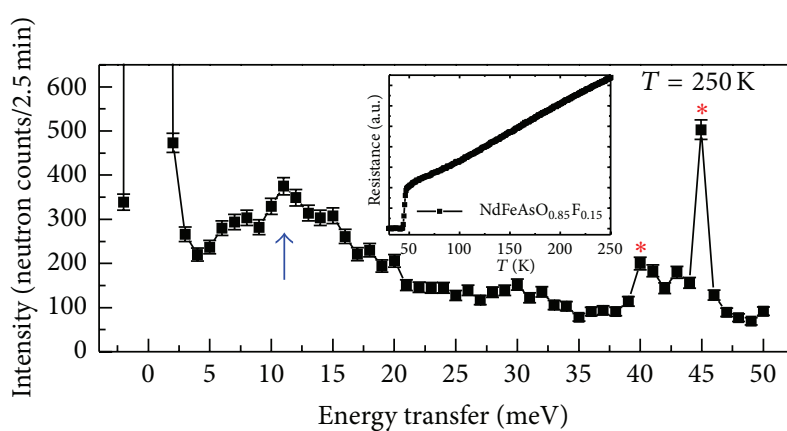

(a)

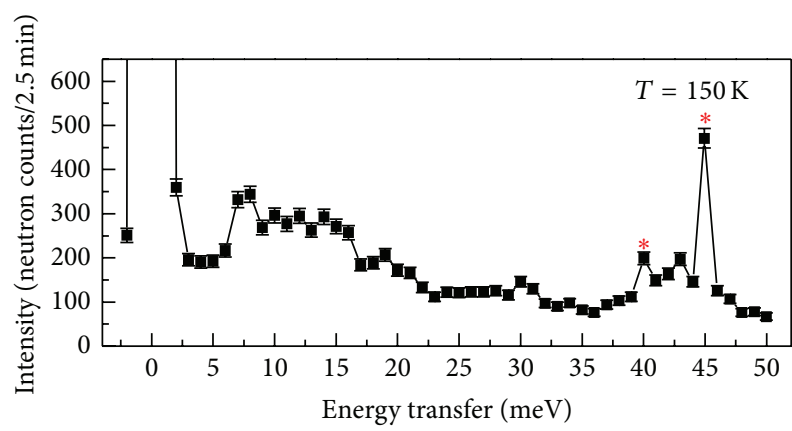

(c)

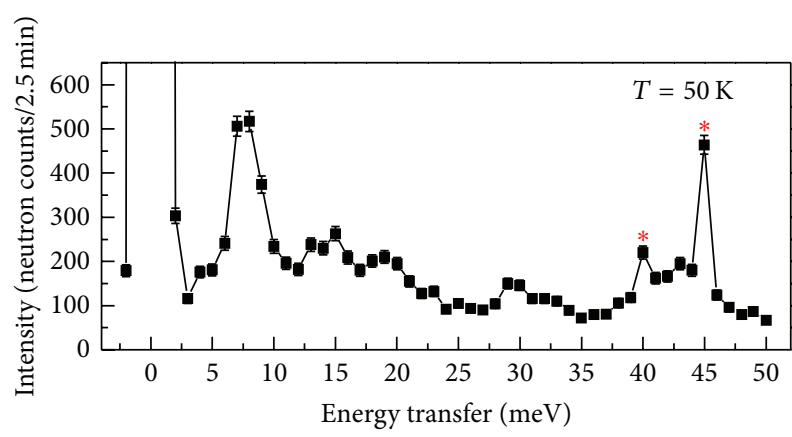

(e)

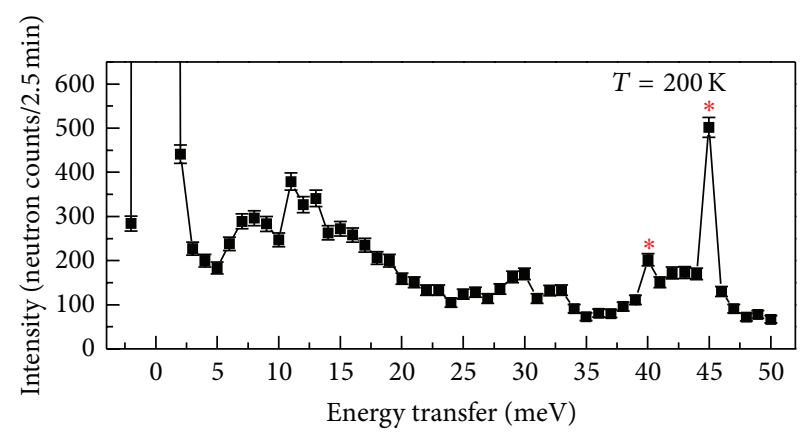

(b)

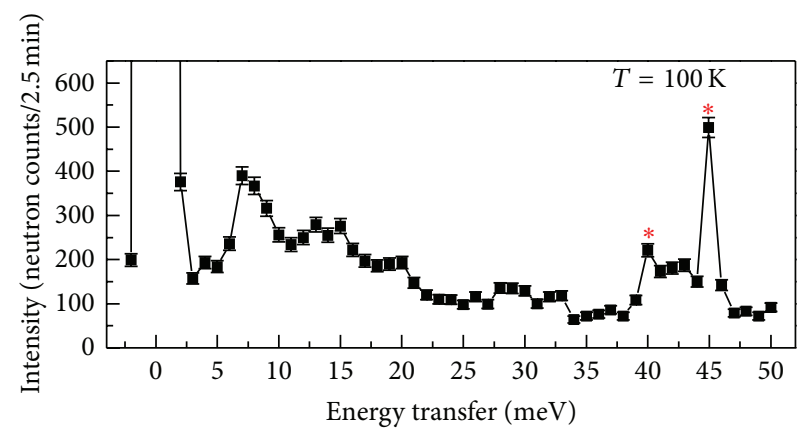

(d)

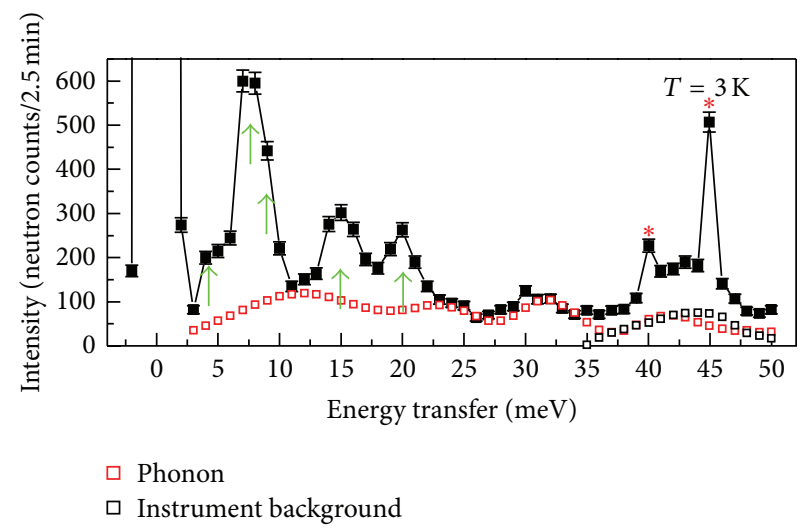

(f)

FIGURE 1: Inelastic neutron scattering spectra of $\mathrm{NdFeAsO}_{0.85} \mathrm{~F}_{0.15}$ were measured with fixed $E_{f}=14.7 \mathrm{meV}$ and $Q=3.1 \AA$. The energy scans were carried out at temperatures from $3 \mathrm{~K}$ to $250 \mathrm{~K}$ as marked on each figure. The solid lines are guides to the eye. The red stars marked the spurious peaks caused by the instrument setup. In the inset of Figure 1(a), the temperature dependence of resistance data of our samples is shown.

the weak crystal field approximation, which applies well to the R elements, the multiplets of different J's (total angular moment) are well separated in energy compared to the intramultiplet splittings; therefore, the interactions between multiplets can be ignored. In such a case, the crystal field states can be considered based solely on the ground-state splitting [17].

According to Hund's rules, the ground state of $\mathrm{Nd}^{3+}$ ion, which contains three f-electrons, is ${ }^{4} I_{9 / 2}$. The splitting of CF levels is directly related to the local lattice symmetry. $\mathrm{NdFeAsO}_{0.85} \mathrm{~F}_{0.15}$ samples have a tetragonal crystal structure with $\mathrm{P} 4 / \mathrm{nmm}$ space group. The Nd atoms are located at the $2 c$ crystallographic site therefore having $C_{4 v}$ point symmetry. This gives the crystal field Hamiltonian as follows:

$$
H_{\mathrm{CEF}}\left(C_{4 v}\right)=B_{2}^{0} O_{2}^{0}+B_{4}^{0} O_{4}^{0}+B_{6}^{0} O_{6}^{0}+B_{4}^{4} O_{4}^{4}+B_{6}^{4} O_{6}^{4},
$$

where $B_{n}^{m}$ are the CF parameters and $O_{n}^{m}$ are Steven's operator equivalents. This $\mathrm{CF}$ symmetry splits the ${ }^{4} I_{9 / 2}$ ground-state multiplet of the $\mathrm{Nd}^{3+}$ ion into five Kramers doublets.

However, suppose the $\mathrm{CF}$ peaks at $T=3 \mathrm{~K}$ in our $\mathrm{NdFe} A s \mathrm{O}_{0.85} \mathrm{~F}_{0.15}$ samples all came from the transitions between the five doublets as in the high temperature tetragonal phase of $\mathrm{NdFeAsO}$ [9]; then the number of groundstate excitation peaks should be at most four. According to 


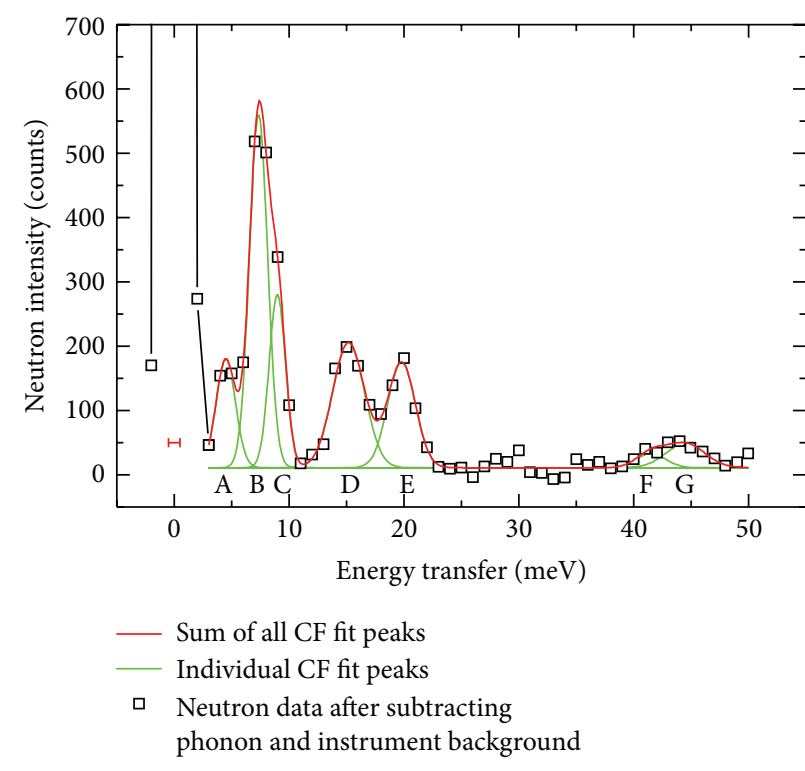

(a)

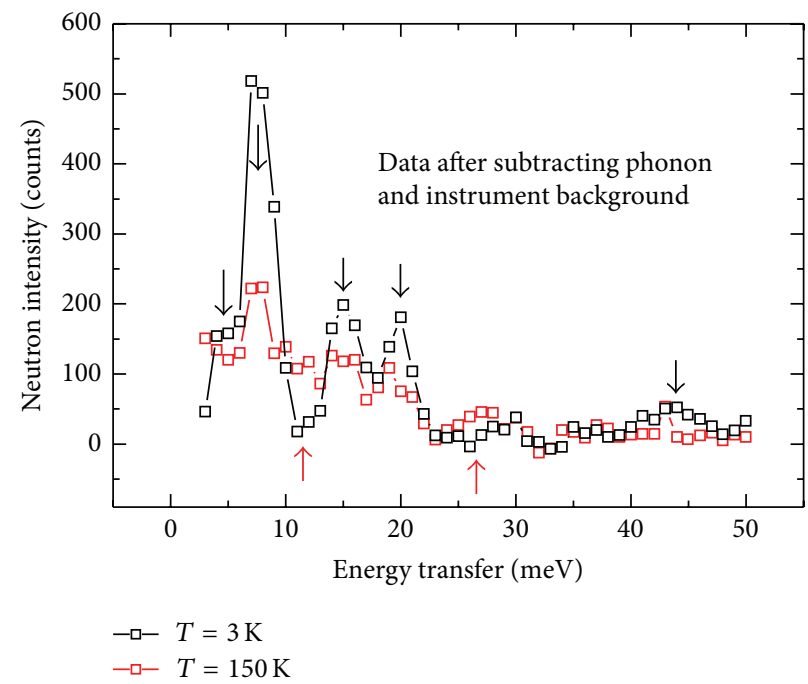

(b)

FIgURE 2: (a) Energy spectra of $\mathrm{CF}$ excitations in $\mathrm{NdFeAs}_{0.85} \mathrm{~F}_{0.15}$ at $\mathrm{T}=3 \mathrm{~K}$ after subtracting the phonon and instrument background. The spectra were well fitted by seven Gaussian peaks. The instrumental energy resolution is $0.98 \mathrm{meV}$ at elastic position (horizontal bar). (b) Comparison of the energy spectra of CF excitations between $T=3 \mathrm{~K}$ and $T=150 \mathrm{~K}$ after subtracting the phonon and instrument background. The black lines are guides to the eye.

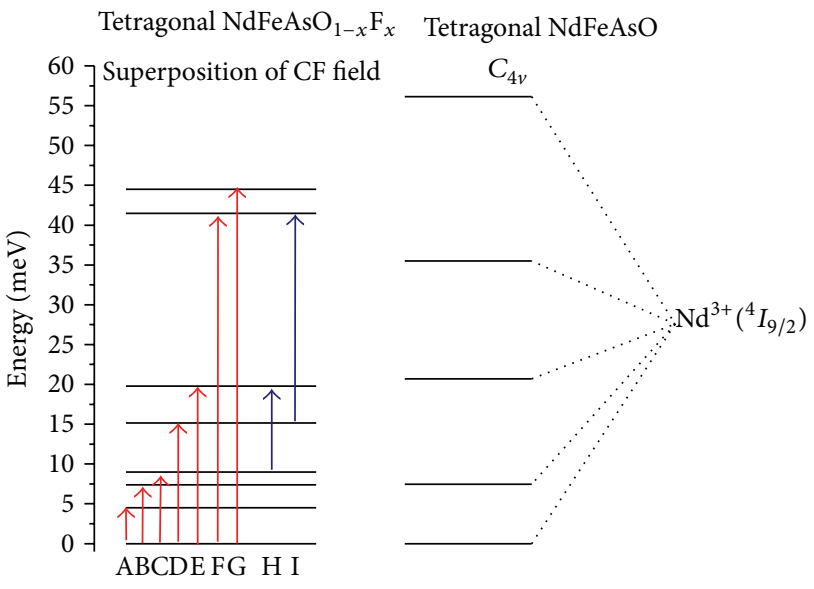

Figure 3: The $\mathrm{Nd}^{3+} \mathrm{CF}$ energy level schemes for both $\mathrm{NdFeAsO}$ and $\mathrm{NdFeAsO} \mathrm{O}_{0.85} \mathrm{~F}_{0.15}$ are shown. The values of energies of $\mathrm{Nd}^{3+}$ in $\mathrm{NdFeAsO}_{0.85} \mathrm{~F}_{0.15}$ are sorted in increasing order: 0, 4.49, 7.34, $8.97,15.17,19.79,41.50$, and $44.51 \mathrm{meV}$. The energies of CF levels of $\mathrm{NdFeAsO}$ were obtained from another paper [9].

our data, the observed CF transitions for $\mathrm{NdFeAsO}_{0.85} \mathrm{~F}_{0.15}$ outnumbered the transitions allowed for $\mathrm{Nd}^{3+}$ with $C_{4 v}$ point symmetry. Therefore the Hamiltonian in (2) is insufficient to describe the CF states in F-doped samples.

A reliable interpretation of the experimental data would be the occurrence of a superposition of crystal fields due to different local symmetries around the $\mathrm{Nd}^{3+}$ ions induced by the fluorine doping, similar to the case of oxygen defects in the $\mathrm{R}$ compounds $\mathrm{RBa}_{2} \mathrm{Cu}_{3} \mathrm{O}_{x}$ [18] and $\mathrm{RCoO}_{x}$ [19]. There are four nearest-neighboring oxygen ions around the $\mathrm{Nd}^{3+}$ ion; the ways of substitution of fluorine for oxygen could not all be the same. So different local charge environments and $\mathrm{CF}$ potentials around $\mathrm{Nd}^{3+}$ ions are expected. As in the analysis of $\mathrm{RCoO}_{x}$ [19], in this situation because of the lowered point symmetry, additional terms are needed to enter the Hamiltonian in (2) which would produce more CF states as in our experimental data. Although it is difficult to give a parameterization in terms of the current complex CF Hamiltonian based on our data, the clear observation of seven ground-state CF transitions would provide strong evidence for the above analysis.

Besides $\mathrm{NdFeAsO}_{0.85} \mathrm{~F}_{0.15}$, the additional $\mathrm{CF}$ states induced by F-doping were also observed in $\mathrm{PrFeAsO}_{0.87} \mathrm{~F}_{0.13}$ [8]. However it is interesting to mention that, for another typical FeAs-1111 compound $\operatorname{CeFeAs}(\mathrm{O}, \mathrm{F})$ [7], there is not much difference between the $\mathrm{CF}$ states in tetragonal $\mathrm{CeFeAsO}$ phase and $\mathrm{PrFeAsO}_{0.86} \mathrm{~F}_{0.14}$ phase. The reason could be that the line widths of major CF transitions in the $\mathrm{Ce}$ compounds are of the order of $10 \mathrm{meV}$, which is significantly larger than that in $\mathrm{Nd}$ compounds (mainly below $3 \mathrm{meV}$ ). So the presumably small effect of fluorine doping could not be detected.

\section{Concluding Remarks}

Inelastic neutron scattering measurements were carried out on polycrystalline samples of $\mathrm{NdFeAsO}_{0.85} \mathrm{~F}_{0.15}$ at different temperatures. Through the analysis of inelastic neutron spectra, up to seven ground-state CF excitations were identified. A crystal field energy level scheme for $\mathrm{Nd}^{3+}$ was proposed 
which shows quite different features comparing with that in tetragonal NdFeAsO phase. This indicates the occurrence of superposition of crystal fields due to the inhomogeneous ligand charge environments and lowered local point symmetries around the $\mathrm{Nd}^{3+}$ upon fluorine doping.

\section{Conflict of Interests}

The authors declare that there is no conflict of interests regarding the publication of this paper.

\section{Acknowledgments}

The authors appreciate the useful discussions with R. Osborn, Songxue Chi, Ninghua Tong, Huining Dong, Tianlong Xia, and Yinguo Xiao. This work is supported by NSFC (no. 11204373), the Fundamental Research Funds for the Central Universities, and the Research Funds of Renmin University of China.

\section{References}

[1] D. R. Temprano, J. Mesot, S. Janssen et al., "Large isotope effect on the pseudogap in the high-temperature superconductor $\mathrm{HoBa}_{2} \mathrm{Cu}_{4} \mathrm{O}_{8}$," Physical Review Letters, vol. 84, no. 9, pp. 1990 1993, 2000.

[2] Y. Kagan, K. A. Kikoin, and A. S. Mishchenko, "Interplay between heavy fermions and crystal-field excitation in Kondo lattices: Low-temperature thermodynamics and inelastic neutron scattering spectra of CeNiSn," Physical Review BCondensed Matter and Materials Physics, vol. 55, no. 18, pp. 12348-12362, 1997.

[3] Y. Kamihara, T. Watanabe, M. Hirano, and H. Hosono, "Ironbased layered superconductor $\mathrm{La}\left[\mathrm{O}_{1-x} \mathrm{~F}_{x}\right] \mathrm{FeAs}(x=0.05-0.12)$ with $T_{c}=26 \mathrm{~K}$," Journal of the American Chemical Society, vol. 130, no. 11, pp. 3296-3297, 2008.

[4] Z. A. Ren, J. Yang, W. Lu et al., "Superconductivity at $52 \mathrm{~K}$ in iron-based F-doped layered quaternary compound $\operatorname{Pr}[\mathrm{O} 1-$ xFx]FeAs," Materials Research Innovations, vol. 12, article 105, 2008.

[5] Z. A. Ren, J. Yang, W. Lu et al., "Superconductivity in the ironbased F-doped layered quaternary compound $\mathrm{Nd}\left[\mathrm{O}_{1-x} \mathrm{~F}_{x}\right] \mathrm{FeAs}$," EPL (Europhysics Letters), vol. 82, no. 5, Article ID 57002, 2008.

[6] Z. A. Ren, W. Lu, J. Yang et al., "Superconductivity at $55 \mathrm{~K}$ in iron-based F-doped layered quaternary compound $\mathrm{Sm}\left[\mathrm{O}_{1-x} \mathrm{~F}_{x}\right]$ FeAs," Chinese Physics Letters, vol. 25, no. 6, pp. 2215-2216, 2008.

[7] S. X. Chi, D. T. Adroja, T. Guidi et al., "Crystalline electric field as a probe for long-range antiferromagnetic order and superconducting state of $\mathrm{CeFeAsO}_{1-x} \mathrm{~F}_{x}$," Physical Review Letters, vol. 101, no. 21, Article ID 217002, 2008.

[8] E. A. Goremychkin, R. Osborn, C. H. Wang et al., "Spatial inhomogeneity in $R \mathrm{FeAsO}_{1-x} \mathrm{~F}_{x}(R=\mathrm{Pr}, \mathrm{Nd})$ determined from rare-earth crystal-field excitations," Physical Review B, vol. 83, no. 21, Article ID 212505, 2011.

[9] Y. Xiao, M. Zbiri, R. A. Downie, J.-W. G. Bos, T. Brueckel, and T. Chatterji, "Inelastic neutron scattering study of crystal field excitations of $\mathrm{Nd}^{3+}$ in NdFeAsO," Physical Review B, vol. 88, Article ID 214419, 2013.

[10] S. A. Danilkin and M. Yethiraj, "TAIPAN: thermal triple-axis spectrometer," Neutron News, vol. 20, no. 4, pp. 37-39, 2009.
[11] R. Osborn, S. Rosenkranz, E. A. Goremychkin, and A. D. Christianson, "Inelastic neutron scattering studies of the spin and lattice dynamics in iron arsenide compounds," Physica C, vol. 469, no. 9-12, pp. 498-506, 2009.

[12] J. Noffsinger, F. Giustino, S. G. Louie, and M. L. Cohen, "Role of fluorine in the iron pnictides: phonon softening and effective hole doping," Physical Review Letters, vol. 102, no. 14, Article ID 147003, 2009.

[13] A. D. Christianson, M. D. Lumsden, O. Delaire et al., "Phonon density of states of $\mathrm{LaFeAsO}_{1-x} \mathrm{~F}_{x}$," Physical Review Letters, vol. 101, no. 15, Article ID 157004, 2008.

[14] L. Soderholm, C.-K. Loong, and S. Kern, "Inelastic-neutronscattering study of the $\mathrm{Er}^{3+}$ energy levels in $\mathrm{ErBa}_{2} \mathrm{Cu}_{3} \mathrm{O}_{7}$," Physical Review B, vol. 45, Article ID 10062, 1992.

[15] E. A. Goremychkin and R. Osborn, "Crystal field excitations in $\mathrm{YbT}_{2} \mathrm{Si}_{2}(\mathrm{~T}=\mathrm{Fe}, \mathrm{Co}, \mathrm{Ni})$," Journal of Applied Physics, vol. 87, no. 9, pp. 6818-6820, 2000.

[16] Y. Qiu, W. Bao, Q. Huang et al., "Crystal structure and antiferromagnetic order in $\mathrm{NdFeAsO}_{1-x} \mathrm{~F}_{x}(x=0.0$ and 0.2$)$ superconducting compounds from neutron diffraction measurements," Physical Review Letters, vol. 101, no. 25, Article ID 257002, 2008.

[17] L. Soderholm, C.-K. Loong, G. L. Goodman, and B. D. Dabrowski, "Crystal-field splittings and magnetic properties of $\mathrm{Pr}^{3+}$ and $\mathrm{Nd}^{3+}$ in $\mathrm{RBa}_{2} \mathrm{Cu}_{3} \mathrm{O}_{7}$," Physical Review $B$, vol. 43 , no. 10, pp. 7923-7935, 1991.

[18] J. Mesot, P. Allenspach, U. Staub, A. Furrer, and H. Mutka, "Neutron spectroscopic evidence for cluster formation and percolative superconductivity in $\mathrm{ErBa}_{2} \mathrm{Cu}_{3} \mathrm{O}_{x}$," Physical Review Letters, vol. 70, no. 6, pp. 865-868, 1993.

[19] A. Furrer, A. Podlesnyak, M. Frontzek et al., "Crystal-field interaction and oxygen stoichiometry effects in strontiumdoped rare-earth cobaltates," Physical Review B, vol. 90, no. 6, Article ID 064426, 2014. 

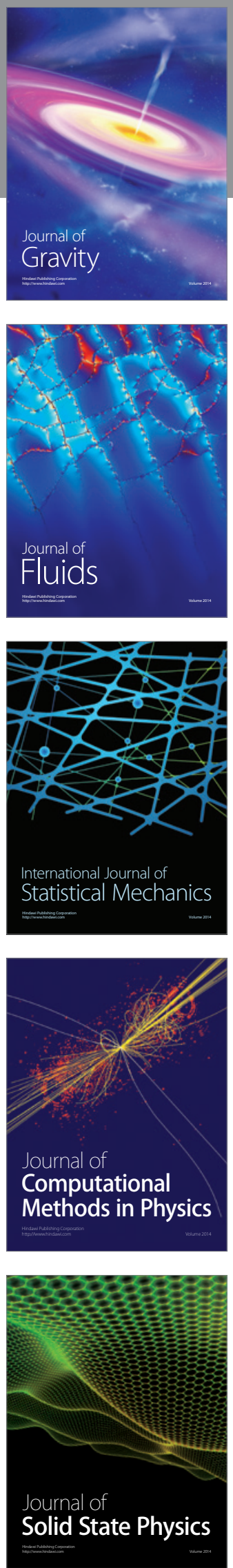

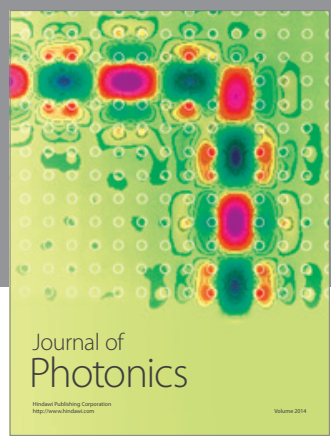

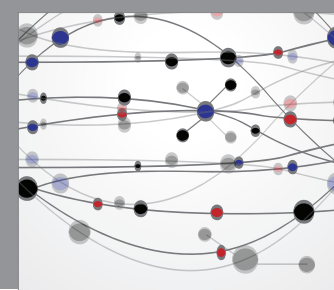

The Scientific World Journal

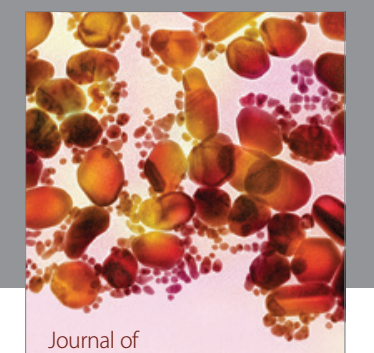

Soft Matter
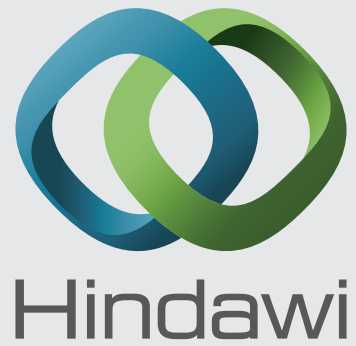

Submit your manuscripts at

http://www.hindawi.com
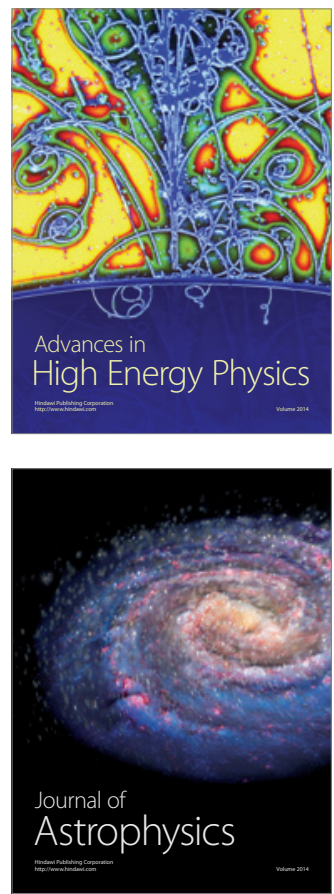
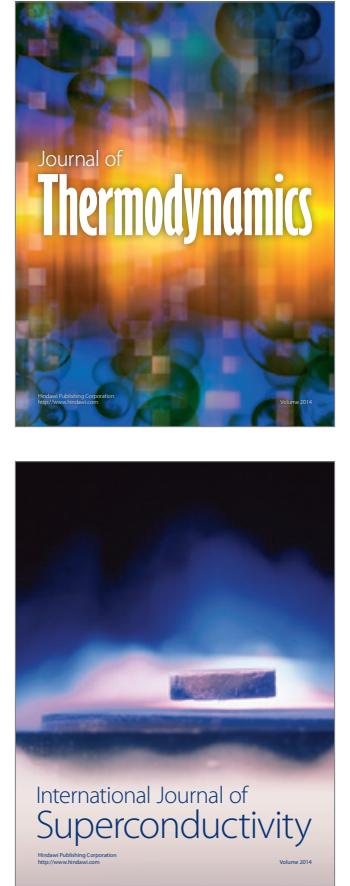
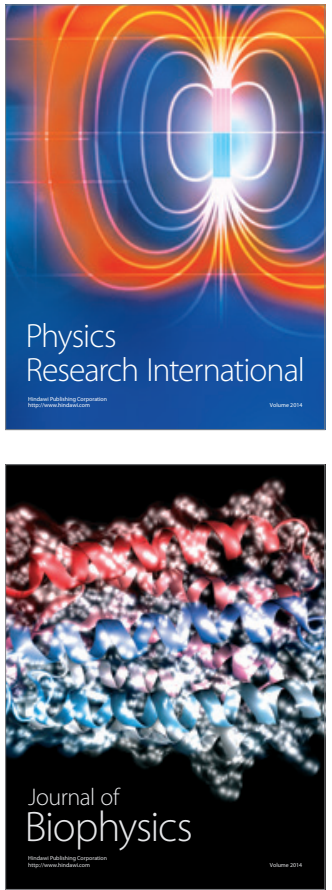
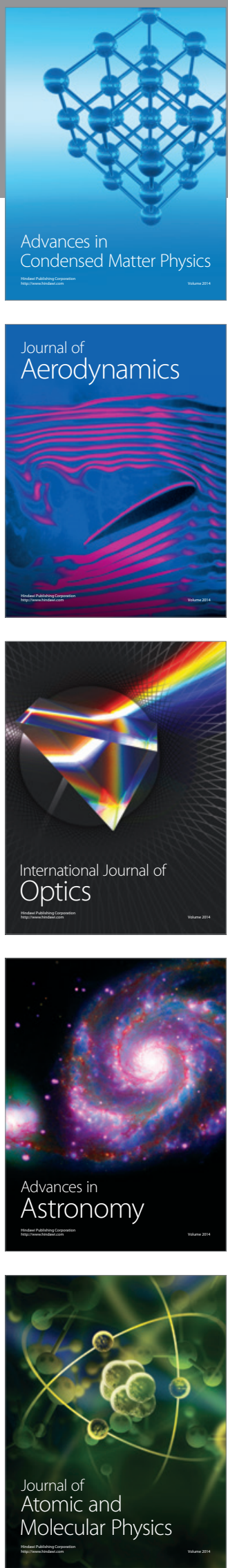